\title{
基于分形的心电信号复杂度及其非线性动力学机制
}

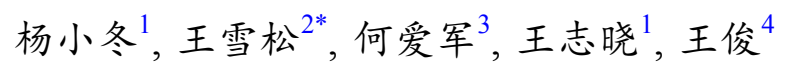

1. 中国矿业大学计算机科学与技术学院, 徐州 221116 ;

2. 中国矿业大学信息与控制工程学院, 徐州市人工智能与大数据重点实验室, 徐州 221116;

3. 南京大学电子科学与工程学院, 南京 210023 ;

4. 南京邮电大学地理与生物信息学院, 南京 210023

* 联系人, E-mail: wangxuesongcumt@163.com

2019-03-19 收稿, 2019-05-17 修回, 2019-05-20 接受, 2019-07-16 网络版发表

国家自然科学基金(61772532，61876186)、江苏省重点研发计划(BE2016773)和江苏省高校自然科学研究重大项目(16KJA310002)资助

摘要 体表心电图(electrocardiogram, ECG)信号是典型的非平稳谐和变频信号, 属于非线性信号.传统的线性分析 方法并不能很好地揭示ECG信号非线性本质。本研究采用多重分形理论, 研究了大量样本同步十二导联心电图信 号的奇异谱面积, 经方差分析 (analysis of variance, ANOVA)检验, 该参数对所研究人群各导联均统计可分. 结果显 示, 健康年轻人(healthy young, HY) 十二导联ECG信号奇异谱面积的算术均值最大、离散度最小, 而心梗(myocardial infraction, MI)患者十二导联ECG信号奇异谱面积算术均值最小、离散度最大, 其他人群如心肌缺血(ischemia) 患者、健康老年人(healthy old, HO)这两个值均处于中等大小水平. 表明随着病变程度加深, 心脏组织类分形结构 受损或者发生结构改变, 导致心电系统非线性动力学复杂程度降低, 同时增加了心电信号传播的不规则性和各向 异性. 另外研究发现, ECG信号奇异谱面积在一定程度上反映了人体自主神经控制的强弱. 随着年龄增长, 奇异谱 面积的十二导联均值逐渐减小, 提示自主神经的自律控制功能逐渐减弱; ECG信号非线性复杂性下降, 由多重分形 趋向单重分形, 意味着个体适应能力的降低.

关键词心电图, 多重分形奇异谱, 算术均值, 离散度

生物医学信号的非线性动力学分析，是目前国内 外研究的热点, 相比于传统线性、时频分析方法能更 好地揭示生命活动本质 ${ }^{[1]}$. 传统的信号分析理论是建 立在傅里叶变换(Fourier transformation, FT)基础上的, 对周期性、平稳信号的分析非常有效. 但傅里叶变换 作为一种全局变换, 并不具备自适应性, 不能很好地反 应信号局部时变特征. Gabor变换(短时傅里叶变换)用 来分析分段平稳信号, 但当信号变化剧烈时, 不能同时 兼顾频率与时间分辨率要求. 小波变换(wavelet transformation, WT)虽然能聚焦到信号任意细节，但对于幅 度较小的频率分量, 跳变时间点描述不够准确. S变换
窗函数宽度随频率呈反向变化, 因而在高频段可获得 较高的时间分辨率, 但同时降低了频率分辨率, 高频处 精确度较差. 维格纳-威利分布(Wegener-Willie distribution, WVD)虽然具有很高的时频分辨率, 但由于其时频 分布函数不是线性变换，对多分量信号会产生严重的 交叉项干扰. 希尔伯特-黄变换(Hilbert-Huang transformation, HHT)能够自适应地产生信号“基”函数, 适用于 非线性、非平稳信号的分析，但其完备性还需要严密 的数学证明，另外其端点效应、包络问题等也需要更 好的方法来解决 ${ }^{[2]}$.

已有非线性动力学参数, 如关联维 $\left(\mathrm{D}_{2}\right)^{[3]}$ 、李雅谱 
诺夫(Lyapunov)指数 ${ }^{[4]}$ 、熵 ${ }^{[5,6]}$ 、多重分形奇异谱 $(f(\alpha))^{[7]}$ 以及多重分形消除趋势波动分析(multifractal detrended fluctuation analysis, MF-DFA) $)^{[8 \sim 11]}$ 等, 已成功地应用于 心电信号研究, 证明其具有混沌特性. 这些非线性动力 学参数比上述时频分析方法在区分确定性“随机”信号 上更为有效. 另外一些文献[12 19]尝试从复杂网络的 角度, 将一维时间序列转换成图、有向或无向网络, 从 而采用网络的量化参数研究时域信号, 取得了很多有 意义的结果. 多重分形奇异谱作为一种重要的非线性 方法, 以往的研究多集中在宽度 $(\Delta \alpha)$ 、非对称性 $(\Delta f)$ 以 及峰值位置分析上 ${ }^{[20 ~ 22]}$. 这种方法描述的是分形体内 最大和最小子集概率测度的差异，反映了整个分形结 构概率测度分布的不均匀程度. 文献[23]定量研究了 MIT-BIH数据库 ${ }^{[24]}$ 中几类正常窦性心律(normal sinus rhythm，NSR)和异常心电图的多重分形奇异谱几何形 状、奇异性指数 $\alpha$ 及 $f(\alpha)$ 取值范围的差异，用于心脏疾 病的无创诊断. 我们研究认为, 相较于一维的 $\Delta \alpha$, 既然 数据序列出现的所有概率都能在多重分形奇异谱曲线 上找到相应的点, 那么该谱曲线所形成的面积也一定 包含了数据序列的所有信息. 这是一种二维的概念, 比 一维分析更能真实地反映信号的非线性本质 ${ }^{[25]}$. 对于 心电图(electrocardiogram, ECG)信号, 其多重分形奇异 谱面积也就包含了心跳非线性动力学的所有信息. 考 虑到心脏组织空间分布的不均匀性和心电信号传播的 各向异性, 本文详细研究了不同人群、大量样本 $E C G$ 信号同步 12 个导联(传感器位置不同)多重分形奇异谱 面积的算术均值和离散度. 结果表明, 这两个参数与心 电系统非线性以及生理、病理活动状态密切相关, 并 对各类人群均统计可分. 研究心电信号的非线性动力 学特性, 进一步掌握其在内部与外界环境影响下, 以及 随着生物体年龄、疾病和自主神经自律控制的变化规
律, 在临床上具有重要的诊断意义.

图1给出一名健康年轻人(healthy young, HY)和一 名典型冠心病(coronary heart disease, CHD)患者ECG信 号的概率密度函数 (probability density function, PDF)曲 线. 从形状看, 该PDF曲线呈现出不同的非对称高斯分 布, 但经量化分析, 信号的均值和标准差分别为 $(-0.0706 \pm 0.1142) \mathrm{mV}(\mathrm{HY})$ 和 $(-0.0802 \pm 0.2862) \mathrm{mV}$ (CHD), 并无明显差异. 因此, 简单的线性参数并不能 有效区分两类人群.

\section{1 理论}

\section{1 多重分形}

先对有关理论作一下简述 ${ }^{[26]}$. 多重分形是用一个谱 函数描述分形体不同层次特性, 从系统的局部出发来研 究其整体规律. 使用Chhabra和Jensen ${ }^{[27]}$ 提出的标准盒计 数法直接计算多重分形奇异谱 $f(\alpha)$, 用尺寸为 $L$ 的盒子覆 盖实验的测度, 计算测度在每个盒中的概率. 那么根据 奇异谱对应于它的奇异强度分布, 多重分形公式被发展 成用来描述一些测度的统计特性和概率分布.

定义测度值为 $T_{i}$ 的段的概率, 即奇异测度 ${ }^{[28]}$ 为

$P_{i}(L)=\frac{T_{i}}{\sum_{i=1}^{N} T_{i}} \sim L^{\alpha_{i}}, \alpha_{i}=\lim _{L \rightarrow \infty} \frac{\ln \left[P_{i}(L)\right]}{\ln L}$,

其中, $T_{i}$ 是某测度被分成 $N$ 等份的时间序列中第 $i$ 份(盒), 本文以每段中电压的平均值作为该段测度值. 指数 $\alpha_{i}$ 称 为奇异强度，反映的是分形体内各小区间奇异性程度. 若奇异强度值在 $\alpha$ 和 $\alpha+\Delta \alpha$ 之间的 $P_{i}(L)$ 有 $N(\alpha)$ 个小区间, 则根据关系 $N(\alpha) \sim L^{-f\left(\alpha_{i}\right)}, f(\alpha)$ 就是具有奇异强度值为 $\alpha$ 的一系列区间段(子集)的分形维数，用豪斯多夫(Hausdorff)维数 ${ }^{[28]}$ 表示. 由此, 建构在盒中概率重整化测度
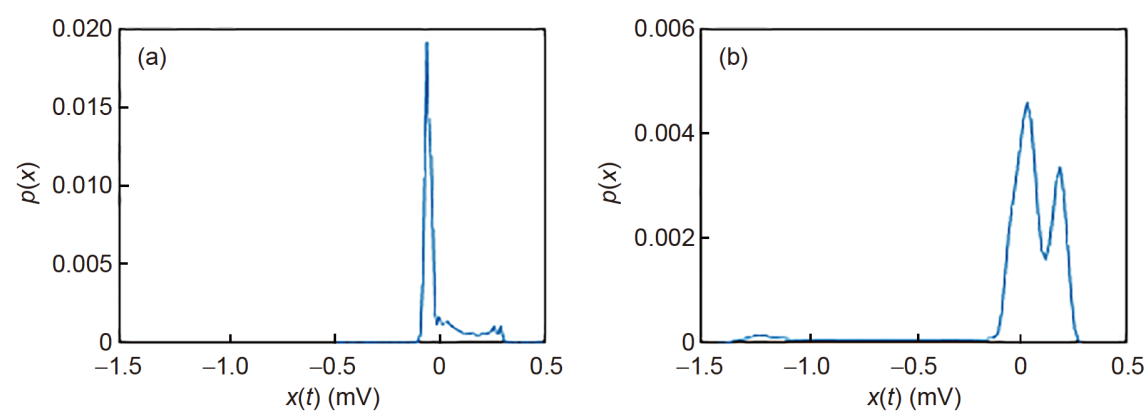

图 1 (网络版彩色)ECG信号的概率密度函数曲线. (a) 健康年轻人; (b) CHD患者

Figure 1 (Color online) PDF curves of the ECG signal. (a) Healthy young; (b) CHD sufferer 
的单参量簇可表示为

$$
\mu_{i}(q, L)=\frac{\left[P_{i}(L)\right]^{q}}{\sum_{i=1}^{N}\left[P_{i}(L)\right]^{q}},
$$

其中, $L$ 定义为 $N^{-1}$.

在一般维数 $D_{q}$ 的定义中, 参量 $q$ 提供了对奇异测度 不同区域的微观探测, 又称权重因子. 对 $q>1, \mu_{i}(q)$ 放大 测度 $P_{i}$ 的强奇异性区域; 对 $q<1, \mu_{i}(q)$ 强调测度的弱奇异 性区域; 而对于 $q=1, \mu(1)$ 则复制原来的测度. 那么, $\mu_{i}(q)$ 理论测度支架的Hausdorff维数 $f(q)$ 由下式给出:

$f(q)=\lim _{L \rightarrow \infty} \frac{\sum_{i=1}^{N} \mu_{i}(q, L) \ln \left[\mu_{i}(q, L)\right]}{\ln L}$.

另外, 关于 $\mu_{i}(q)$ 奇异强度 $\alpha_{i}=\ln P_{i} / \ln L$ 的平均值可由下式 计算:

$\alpha(q)=\lim _{L \rightarrow \infty} \frac{\sum_{i=1}^{N} \mu_{i}(q, L) \ln \left[P_{i}(L)\right]}{\ln L}$.

式(3)和(4)给出了作为参量 $q$ 函数的Hausdorff维数 $f(\alpha)$ 和平均奇异强度 $\alpha$ 之间的关系. 奇异谱曲线 $f(\alpha)-\alpha$ 在 $\alpha$ 轴 分布区域(宽度)的大小表示多重分形强弱, 大的 $\Delta \alpha$ 值与 强的多重分形相联系，小的 $\Delta \alpha$ 代表弱的多重分形并趋 于单重分形.

描述多重分形的另外一组参量是 $D_{q}-q$. 定义 $q$ 阶配 分函数:

$\chi_{q}(L)=\sum_{i=1}^{N}\left[P_{i}(L)\right]^{q}=\sum_{i=1}^{N} L^{\alpha_{i} q} \sim L^{\tau(q)}$,

其中, $\tau(q)$ 称为质量指数, $q$ 为权重因子:

$\tau(q)=\lim _{L \rightarrow \infty} \frac{\ln \chi_{q}(L)}{\ln L}$

相对于概率测度 $q$ 阶矩的伸缩指数，一般维 $D_{q}(q$ 次信息 维、广义分维)提供了对奇异测度的另一种描述. 它被 定义为

$$
D_{q}=\left\{\begin{array}{l}
\lim _{L \rightarrow \infty} \frac{1}{q-1} \frac{\ln \chi_{q}(L)}{\ln L} \\
=\frac{1}{q-1} \lim _{L \rightarrow \infty} \frac{\ln \sum\left[P_{i}(L)\right]^{q}}{\ln L}, q \neq 1 . \\
\lim _{L \rightarrow \infty} \frac{\sum P_{i}(L) \ln \left[P_{i}(L)\right]}{\ln L}, q=1 .
\end{array}\right.
$$

实际上, $D_{q}$ 是通过空间各子集奇异测度的 $q$ 次方及其求 和运算，从整体上反映各子集奇异性程度的. 因此，具 有不同标度指数的子集通过 $q$ 值改变得以区分，也就是
把一个分形分成许多具有不同奇异程度的区域来研究, 以便于分层次了解分形体内部结构. 可以证明： $\lim _{q \rightarrow-\infty} D_{q}=\alpha_{\text {max }}, \lim _{q \rightarrow+\infty} D_{q}=\alpha_{\text {min }} . \alpha_{\text {max }}, \alpha_{\text {min }}$ 分别是最大、最 小奇异性指数.

$f(\alpha)$ 和 $D_{q}$ 分别是 $\alpha$ 和 $q$ 的函数. $f(\alpha)$ 由式 $\tau(q)=(q-1) D_{q}$ $(L \rightarrow 0)$ 与 $D_{q}$ 相联系, 该公式由勒让德变换决定, 即 $f(\alpha)$ $=q \alpha(q)-\tau(q), \alpha(q)=\mathrm{d}[\tau(q)] / \mathrm{d} q$.

图2(a)是图1(b)所讨论CHD患者的 $\mathrm{V}_{2}$ 导联ECG信 号时域波形. 依据1.1的理论分别作出该导联对应的几 种多重分形曲线, 如图2(b) (f)所示. 其中图2(b)是其多 重分形奇异谱 $f(\alpha)-\alpha$, 沿 $\alpha$ 轴的宽度 $\Delta \alpha=\alpha_{\text {max }}-\alpha_{\text {min }} . \Delta \alpha$ 越 大, 说明信号的非线性复杂度越高. 根据式(3), 作出图2 (c), 参量 $q$ 自上到下分别取值-10, $-5,5$. 图中直线表 明 $\sum \mu_{i} \ln \mu_{l}$ 与 $\ln L$ 是成正比的, 比例 $f$ 的值与区间划分无关 $(L \rightarrow 0)$, 每条曲线的斜率分别对应不同的 $f$ 值, 最后相交 于点 $(0,0)$, 因此证明该数据序列具有多重分形特性. 图 2 (d) 为式(6)得到的双对数坐标下不同 $q$ 值的质量配分 函数曲线, 图中 $q$ 自上至下分别取值 $-7 \sim 7$, 间隔为 2 , 各 曲线的斜率即分别对应不同的质量指数 $\tau(q)$. 图 $2(\mathrm{e})$ 为 其广义维数 $D_{q}$ 随 $q$ 的变化曲线(式(7)), $D_{q}$ 沿纵轴的宽度 即为 $\Delta \alpha$, 反映了信号波动的大小. 图2(f)是该导联ECG 信号的质量指数谱 $\tau(q)-q$ 曲线, 其弯曲程度(斜率)常作 为多重分形复杂度的衡量指标，图中近似线性表明此 信号来自病理的心跳动力学, 已趋于单重分形.

\section{2 奇异谱面积}

为了更好地量化多重分形谱分析，提出奇异谱面 积的概念. 作为比较, 图3(a)给出奇异谱宽度 $\Delta \alpha$ 的示意 图. 图3(b)中, 采用谱曲线 $f(\alpha)-\alpha$ 首尾连线 $(\mathrm{AB})$ 与谱曲线 所围成的图形的面积, 即 $S_{f(\alpha)}$, 有效避免了因曲线位置 移动带来的偏差，能更好地反映原始信号的非线性本 质 ${ }^{[25]}$.

$$
\begin{array}{r}
S_{f(\alpha)} \text { 由下式表达: } \\
S_{f(\alpha)}=\int f(\alpha) \mathrm{d} \alpha-S 1,
\end{array}
$$

其中, $S 1$ 为谱曲线首尾连线与 $\alpha$ 轴所形成的多边形面积. $S_{f(\alpha)}$ 的意义可以这样理解: $f(\alpha)$ 和 $\alpha$ 共同构成了测度数据 序列的二维空间，所有数据所出现概率的奇异强度和 相应维数都可以从这个空间中找到对应点, 因此 $f(\alpha)$ 和 $\alpha$ 的积分一定包含了该数据序列全部信息. $S_{f(\alpha)}$ 的值越 大, 表明数据序列的非线性动力学复杂程度越高. 

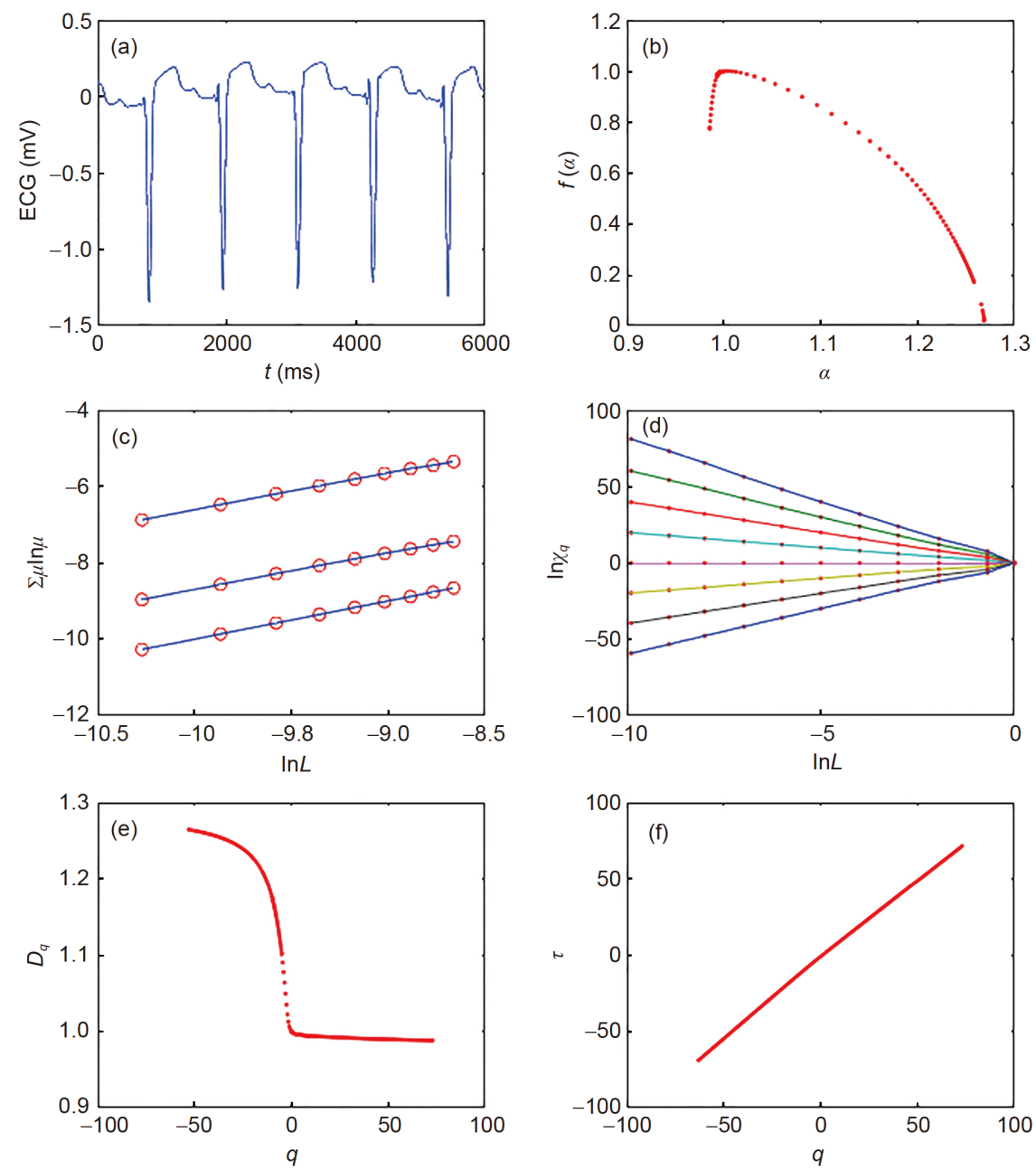

图 2 (网络版彩色)一名典型冠心病 $(\mathrm{CHD})$ 患者 $\mathrm{V}_{2}$ 导联心电图及对应的各种多重分形曲线. (a) ECG信号时域波形; (b) 多重分形奇异谱 $f(\alpha)-\alpha$; (c) $\sum \mu_{i} \ln \mu_{i}-\ln L$ 曲线; (d) 配分函数双对数曲线; (e) 广义维数谱 $D_{q}-q$; (f) 质量指数曲线 $\tau(q)-q$

Figure 2 (Color online) $\mathrm{V}_{2}$ lead's ECG signal and its corresponding multifractal curves for a typical CHD sufferer. (a) The time domain wave; (b) multifractal singularity spectrum $f(\alpha)-\alpha$; (c) curve for $\sum \mu_{i} \ln \mu_{l}-\ln L$; (d) $\log -\log$ plot of the partition function; (e) generalized dimension spectrum $D_{q}-q$; (f) mass exponent curve $\tau(q)-q$
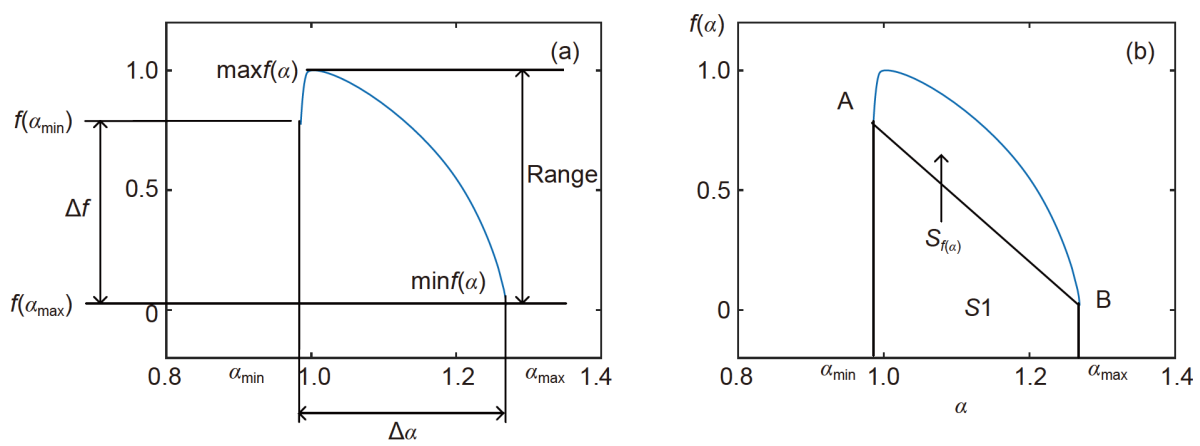

图 3 (网络版彩色)图2(b)中CHD患者ECG信号多重分形奇异谱 $f(\alpha)$ - $\alpha$ 参数的量化分析. (a) 宽度 $\Delta \alpha$ 、非对称性 $\Delta f$ 以及峰值位置; (b) 奇异谱面积 $S_{f(a)}$

Figure 3 (Color online) Quantitation analysis of the multifractal singularity spectrum $f(\alpha)-\alpha$ as for the CHD sufferer shown in Fig. 2(b). (a) Width $\Delta \alpha$, asymmetry $\Delta f$ and the peak position; (b) area of the singularity spectrum $S_{f(a)}$ 
事实上，人的 12 个导联ECG信号多重分形奇异谱 并不是重合在一起，而是离散分布的. 其谱曲线所形成 的面积各不相同，代表了各导联多重分形强弱的差异. 我们用标准差描述各导联谱面积对数的离散程度, 进 一步引人离散度 ${ }^{[29]}$ (即标准差). 该值越大，表明奇异谱 面积相差越大, 即心跳动力学复杂程度相差也越大. 计 算公式如下:

$\overline{\ln S_{f(\alpha)}}=\frac{1}{N} \sum_{i=1}^{N} \ln S_{i}$,

$\delta_{\ln S_{f(\alpha)}}=\sqrt{\frac{1}{N-1} \sum_{i=1}^{N}\left[\ln S_{i}-\overline{\ln S_{f(\alpha)}}\right]^{2}}$,

其中, $\overline{\ln S_{f(\alpha)}}$ 为 12 个导联ECG信号谱面积对数 $\ln S_{f(\alpha)}$ 的 算术平均值, $\delta_{\ln S_{f(\alpha)}}$ 即为其离散度. $N=12$ (12个导联), $S$ 的下标 $i$ 代表导联.

经过对不同人群、不同年龄段 $\overline{\ln S_{f(\alpha)}}, \delta_{\ln S_{f(\alpha)}}$ 参数 的比较, 发现各组人群的这两个参数值并不相同, 它们 与心脏电活动非线性动力学特性密切相关. 本文试图 从不同个体的这种差异，找到区分健康人及有疾病人 的一些规律.

\section{2 实验设计}

\section{1 实验对象}

本实验 $\mathrm{ECG}$ 数据来源于上海长征医院南京分院心 内科 ${ }^{[30]}$. 实验对象分为 $\mathrm{A}, \mathrm{B}, \mathrm{C}$ 三组, 共84例. 其中, $\mathrm{A}$ 组 共27例, 包括脑部受伤(brain injury)患者12例(平均年龄 土标准差: (37.83 \pm 11.13 )岁), 高血压(high blood pressure, HBP)患者15例(平均年龄土标准差: $(60.60 \pm 9.50)$ 岁); B 组为CHD患者组, 共25例, 包括心肌缺血(ischemia)患 者15例(平均年龄土标准差: (63.53 \pm 6.32$)$ 岁), 心梗(myocardial infarction, MI)患者10例(平均年龄土标准差： (58.90土11.48)岁); C组为健康对照组, 共32例, 包括健 康老年人(healthy old, HO)12例(平均年龄土标准差: (62.67 \pm 5.63$)$ 岁, 血压正常, 无心脏病史), 健康年轻人 (healthy young, HY)20例(平均年龄土标准差: (22.50 \pm 3.50 ) 岁, 窦性心率).

\section{2 实验装置和数据处理}

采用南京大学生物医学电子研究所研制的NHE2000 型心电工作站 ${ }^{[31]}$, 可检测和显示 $100 \mathrm{~Hz}$ 以上的高频 成分(100 Hz 2 kHz). 文献[32]报道相较常规心电图(频
率分量大致分布在0.05 100 Hz范围内), 高频心电图 (high frequency ECG, HFECG)频率分量可达0.02 Hz $\sim 2 \mathrm{kHz}$, 甚至一直延伸到 $3 \mathrm{kHz}^{[33]}$, 对冠心病的检出率可 达67\% 90\%, 而常规心电图仅为 $59.43 \%$. 该系统具有频 率响应宽、灵敏度高的特点, $\mathrm{A} / \mathrm{D}$ 转换位数 $12 \mathrm{bit}$, 一个 数据用 2 byte来表示(高4位置 0 ). 受试者保持平躺休息状 态, 在安静条件下几分钟后开始做同步十二导联心电图 信号采集. 体表ECG信号经放大后送人机内专用 $\mathrm{A} / \mathrm{D}$ 采 集卡, 采样频率 $1 \mathrm{kHz}$, 时间长度约为 $1 \mathrm{~min}$, 按不同位置 共分12组(即导联, 每个导联大约采60000个点).

为了更好地分析数据，采集后的心电数据在实际 计算前先经过小波滤波，用的是bior 6.8 小波基函数， 滤去呼吸波(低频)、50 Hz工频干扰噪声(中频)和肌电 干扰(高频). 另外，为了消除信号刚开始采集时的不稳 定状态, 去掉每组数据前 2000 个点, 并把剩下的点按尺 寸 $L=2$ 划分成 $N$ 个区间 $(1 / N \rightarrow 0)$. 设置参数 $q$ 为从 $-\infty$ 到 $+\infty$ 取值, 其步长根据数据点的疏密而变.

\section{3 结果与分析}

\section{1 实验结果}

首先根据式(9)，计算A组中每个实验对象ECG信 号多重分形奇异谱面积 $S_{f(\alpha)}$ 的对数, 并分别对 12 个导 联取算术均值, 然后作 $\overline{\ln S_{f(\alpha)}}$ 与年龄关系图, 如图4所 示. 该图明显显示 $\overline{\ln S_{f(\alpha)}}$ 随年龄增长呈下降趋势. 这表 明随着年龄增长，人体 $\mathrm{ECG}$ 信号的非线性动力学复杂 程度下降, 由多重分形趋向单重分形. 该结论与文献 [34]的描述一致. 进一步研究发现，人的ECG信号多重 分形奇异谱面积在一定程度上反映了人体自主神经 (交感和副交感)自律控制的强弱 ${ }^{[29]}$. 当年龄增长, 该控 制功能也逐渐减弱.

本实验中，总共计算了 84 个样本(包括健康人和有 疾病人)、1008个导联ECG信号的多重分形奇异谱面 积对数, 发现每个个体、不同导联都存在差异, 说明具 有不同的非线性动力学特性和非线性复杂度. 下面对 结果进行统计分析.

表1，2分别是B，C两组中每个导联ECG信号的 $\ln S_{f(\alpha)}$ 值在相同人群中各同名导联的统计平均. 从统计 结果看, 心肌缺血(ischemia)患者各导联多重分形奇异 谱面积均处于中等大小水平. 表明心肌缺血虽然降低 了心脏动力学复杂程度，使得肌体协调控制系统出现 

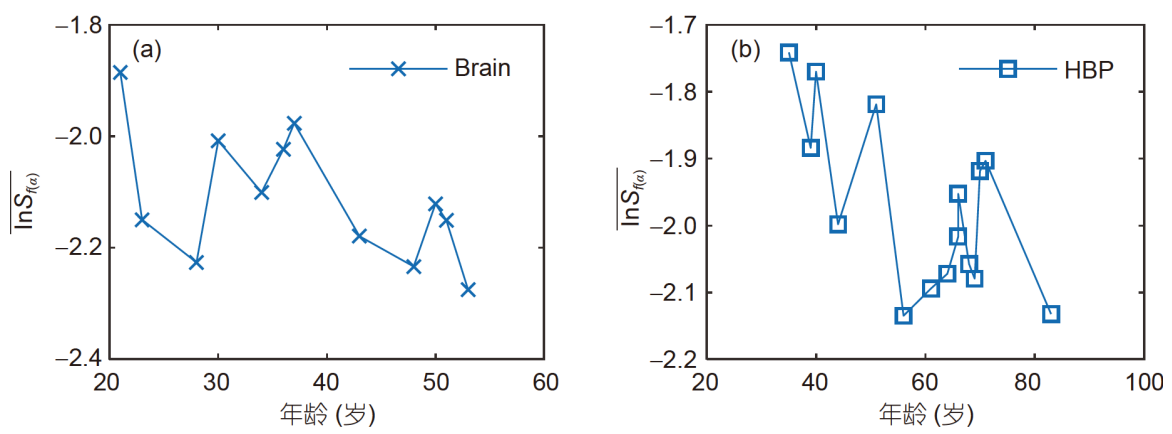

图 4 (网络版彩色)A组ECG信号多重分形奇异谱面积对数的十二导联均值随年龄变化关系. (a) 脑受伤患者; (b) 高血压患者

Figure 4 (Color online) Variation of the 12 leads' mean of multifractal singularity spectrum area logarithm from ECG signals of group A with age. (a) Brain injury sufferer; (b) high blood pressure sufferer

表 1 同步十二导联人体ECG信号多重分形奇异谱面积的对数(肢体导联)

Table 1 Area logarithm of the synchronous 12 leads' multifractal singularity spectrum for ECG signals of group B and group C (limb leads)

\begin{tabular}{ccccccc}
\hline 导联 & I & II & III & AVR & AVL & AVF \\
\hline Ischemia & $-2.16 \pm 0.46$ & $-2.18 \pm 0.29$ & $-2.25 \pm 0.35$ & $-2.26 \pm 0.31$ & $-2.40 \pm 0.48$ & $-2.31 \pm 0.36$ \\
MI & $-2.44 \pm 0.36$ & $-2.58 \pm 0.37$ & $-2.68 \pm 0.62$ & $-2.68 \pm 0.38$ & $-2.90 \pm 0.72$ & $-2.59 \pm 0.25$ \\
HY & $-2.02 \pm 0.21$ & $-1.83 \pm 0.18$ & $-1.87 \pm 0.20$ & $-1.91 \pm 0.11$ & $-2.05 \pm 0.28$ & $-1.86 \pm 0.18$ \\
$P$-value & 0.0075 & 0.0000 & 0.0000 & 0.0000 & 0.0001 & 0.0000 \\
\hline
\end{tabular}

表 2 同步十二导联人体ECG信号多重分形奇异谱面积的对数(胸导联)

Table 2 Area logarithm of the synchronous 12 leads' multifractal singularity spectrum for ECG signals of group B and group C (chest leads)

\begin{tabular}{ccccccc}
\hline 导联 & $\mathrm{V}_{1}$ & $\mathrm{~V}_{2}$ & $\mathrm{~V}_{3}$ & $\mathrm{~V}_{4}$ & $\mathrm{~V}_{5}$ & $\mathrm{~V}_{6}$ \\
\hline Ischemia & $-2.22 \pm 0.46$ & $-1.75 \pm 0.32$ & $-1.75 \pm 0.31$ & $-1.78 \pm 0.32$ & $-1.75 \pm 0.28$ & $-1.85 \pm 0.29$ \\
MI & $-2.09 \pm 0.27$ & $-1.93 \pm 0.23$ & $-2.24 \pm 0.43$ & $-1.95 \pm 0.21$ & $-2.10 \pm 0.41$ & $-2.12 \pm 0.31$ \\
HY & $-1.77 \pm 0.22$ & $-1.64 \pm 0.13$ & $-1.75 \pm 0.18$ & $-1.59 \pm 0.11$ & $-1.61 \pm 0.12$ & $-1.88 \pm 0.17$ \\
$P$-value & 0.0007 & 0.0076 & 0.0001 & 0.0004 & 0.0000 & 0.0226 \\
\hline
\end{tabular}

了某些功能成分上的退化, 但由于程度尚轻, 还没有对 组织造成太大伤害、改变其分形结构. 冠心病中较严 重的心梗(myocardial infraction, MI)患者各导联多重分 形奇异谱面积几乎都明显小于其他人群相同导联的多 重分形奇异谱面积, 提示心肌广泛受损, 严重的心肌缺 血导致ECG信号复杂程度降低. 冠状动脉血管狭窄或 者堵塞, 导致相应组织类分形结构受损, 或者发生结构 变化 ${ }^{[35,36]}$, 这可能是系统的复杂性显著降低、心梗患 者多重分形谱面积普遍小于其他人的原因．健康年轻 人 $(\mathrm{HY})$ 各导联多重分形奇异谱面积几乎都要明显大于 其他人群相应导联的多重分形奇异谱面积，这是由于 健康年轻人心脏处于最佳生理状态，非线性动力学特 性最强的缘故．另外，各导联单因素方差分析(analysis of variance, ANOVA)检验结果也列于表中, 该值接近零 表示至少有一组数据的样本均值与其他两组有显著差 异. 结果显示, 在 $P<0.05$ 显著性水平下, 每一个导联均 能得到有效区分，但肢体导联总体区分效果要好于胸 导联

从这两张表中还可以看出，无论是健康人还是有 疾病人，他们胸部导联(尤其是 $\mathrm{V}_{2} \sim \mathrm{V}_{6}$ 导联)多重分形奇 异谱面积都要大于相应的肢体导联. 这是因为胸部导 联距心脏较近, 信号质量较好, 且肌电干扰小, ECG信 号的非线性动力学复杂程度较强; 而肢体导联距心脏 较远, 测得的ECG信号经过噪声干扰和衰减, 非线性复 
杂程度减弱.

\section{2 进一步研究}

进一步分析, 根据式(9), (10), 首先计算B, C两组每 个个体ECG信号 12 个导联多重分形奇异谱面积对数的 算术均值和离散度(图5), 然后分别在相同人群中进行 统计平均, 记为 $\overline{\overline{\ln S_{f(\alpha)}}}, \overline{\delta_{\ln S_{f(\alpha)}}}$, 结果如表3所示.

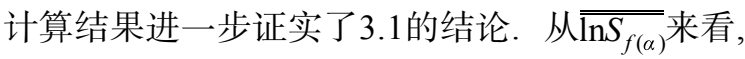
样本的统计数据表明，除心梗患者明显偏小、健康年 轻人明显偏大外, 其他人群大致相等, 均处于中等大小 水平; 从离散度可得出类似结论: 心梗患者的 $\delta_{\ln f_{f(\alpha)}}$ 值 明显大于其他人群，而健康年轻人该值明显小于其他 人群. 说明心梗患者各导联多重分形谱面积相差很大, 即各导联ECG信号的非线性动力学特性有很大差异, 而健康年轻人的这种差异则最小. 这意味着心梗患者 除了心脏组织、冠状动脉和静脉血管树等类分形结构 受损, 系统复杂程度降低外, 同时也增加了其电信号传 播的不规则性和各向异性. 其他人群 $\bar{\delta}_{\operatorname{lns} f_{f(\alpha)}}$ 值无太大 差别. 其中心肌缺血患者处于相对较小的水平, 健康老 年人处于相对较大水平.上述结果如图6所示.

\section{3 参数分类结果检验}

为检验图 6 中两个参数 (算术均值 $\overline{\ln S_{f(\alpha)}}$ 和离散 度 $\delta_{\ln S_{f(\alpha)}}$ 的分类效果, 对它们进行了 $t$ 检验分析. 结果如 下, $\overline{\ln S_{f(\alpha)}}$ 对各人群的 $t$ 检验值分别为 $P_{\mathrm{HY}, \mathrm{Isc}}=0.0018$, $P_{\mathrm{HY}, \mathrm{MI}}=2.8158 \times 10^{-8}, P_{\mathrm{Isc}, \mathrm{MI}}=0.0109 ; \delta_{\mathrm{ln} S_{f(\alpha)}}$ 的 $t$ 检验值为 $P_{\mathrm{HY}, \mathrm{Isc}}=4.5004 \times 10^{-6}, P_{\mathrm{HY}, \mathrm{MI}}=1.8186 \times 10^{-5}, P_{\mathrm{Isc}, \mathrm{MI}}=0.1369$. 其中, 概率 $P$ 的下标代表两组比较的人群.

该结果表明，同样在 $P<0.05$ 的显著性水平下，除参 数 $\delta_{\mathrm{In} S_{f(\alpha)}}$ 的检验值 $P_{\mathrm{Isc}, \mathrm{MI}}=0.1369$ 外, 其他任两组数据均 能得到有效区分. 其中, 算术均值的总体区分效果要好 于离散度.

\section{4 讨论}

心跳动力学中的多重分形现象属于其控制机制中 的内在特性, 这种特性不简单地随其他因素而改变, 如 外部刺激、物理活动的激烈程度以及身体姿态的变化

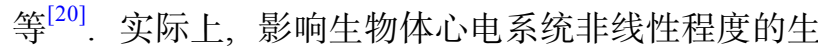

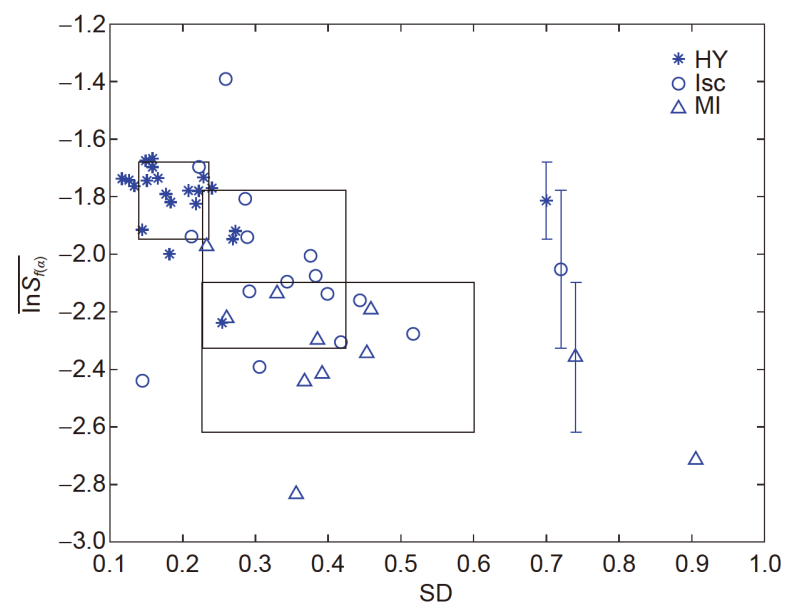

图 5 (网络版彩色) B和C两组人群中不同个体ECG信号的多重分形 奇异谱面积对数的十二导联均值和标准差. 符号分别代表不同的人 群, 矩形则定义为各自的组内均值和标准差

Figure 5 (Color online) The 12 leads' mean and standard deviation (SD) of the multifractal singularity spectrum area logarithm from ECG signals of group B and group C. Symbols represent the different crowds, and rectangles denote the mean and the SD in each corresponding group

表 3 三组人群同步十二导联ECG信号多重分形奇异谱面积对 数的算术均值 $\overline{\ln S_{f(\alpha)}}$ 和离散度 $\delta_{\ln S_{f(\alpha)}}$ 的统计结果

Table 3 Statistics of the mean and the dispersing degree of the 12 leads' multifractal singularity spectrum area logarithm from ECG signals of groups $\mathrm{A}, \mathrm{B}$ and $\mathrm{C}$

\begin{tabular}{lcc}
\hline 实验对象 & $\overline{\overline{\ln S_{f(\alpha)}}}$ & $\overline{\delta_{\ln S_{f(\alpha)}}}$ \\
\hline A组: Brain injury & $-2.0316 \pm 0.2531$ & $0.3592 \pm 0.1008$ \\
HBP & $-2.0202 \pm 0.1100$ & $0.3268 \pm 0.0865$ \\
B组: Ischemia & $-2.0526 \pm 0.2749$ & $0.3259 \pm 0.0985$ \\
MI & $-2.3573 \pm 0.2605$ & $0.4140 \pm 0.1872$ \\
C组: HO & $-2.0890 \pm 0.1628$ & $0.3678 \pm 0.0722$ \\
HY & $-1.8139 \pm 0.1345$ & $0.1878 \pm 0.0482$ \\
\hline
\end{tabular}

理、病理因素有很多, 有自主神经控制引起的, 有心脏 本身电活动及病变引起的, 也有随着年龄增长, 肌体综 合功能减弱引起的. 然而, 对如此复杂的非线性动力学 系统, 并没有确定的数学方程可以描述, 因此通过实验 模拟其复杂行为从而进行研究就显得非常必要.

本文采用非线性理论，研究了不同生理、病理活 动状态下ECG信号的多重分形奇异谱面积，发现各组 人群十二导联均值均随年龄增长呈下降趋势. 分析认 为, 随着年龄增长, 人体自主神经对心肌组织的自律控 制功能逐渐减弱, ECG信号非线性复杂程度降低, 由多 

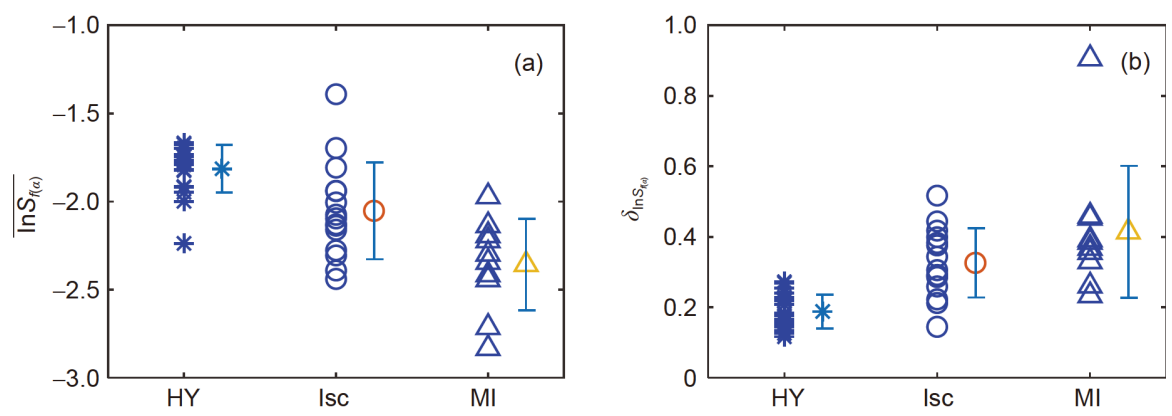

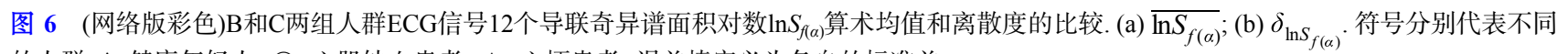
的人群. * 健康年轻人; $\bigcirc$, 心肌缺血患者; $\triangle$, 心梗患者. 误差棒定义为各自的标准差

Figure 6 (Color online) Comparison of $\overline{\ln S_{f(\alpha)}}$ (a) and $\delta_{\ln S_{f(\alpha)}}$ (b) of the 12 leads' multifractal singularity spectrum area logarithm from ECG signals of groups B and C. Symbols represent the different crowds: *, the healthy young; $\bigcirc$, ischemia sufferer; $\triangle$, MI sufferer. The error bars denote the corresponding standard deviation

重分形趋向单重分形. 研究发现, 心梗患者各导联多重 分形奇异谱面积几乎都要明显小于其他人群，而其离 散度最大. 这可能是由于冠状动脉血管狭窄或堵塞, 严 重的心肌缺血导致在某一个或某几个导联对应位置心 脏组织的自相似结构发生变化, 系统的非线性复杂程 度降低，同时也增加了电信号传播的不规则性和各向
异性. 而健康年轻人各导联ECG信号的多重分形奇异 谱面积最大、离散度最小. 表明健康年轻人心脏处于 最佳的生理状态, 非线性动力学复杂程度最高, 其电信 号传播也较均匀. 其他人群(如心肌缺血患者、健康老 年人)多重分形奇异谱面积的算术均值和离散度均处 于中等大小水平.

\section{参考文献}

1 Ning X, Bian C, Wang J, et al. Research progress in nonlinear analysis of heart electric activities. Chin Sci Bull, 2006, 51: 385-393

2 Zhang M J, Li S G, Sun Q L. Research and simulation on four typical methods of time-frequency analysis theory (in Chinese). Instrum Anal Monit, 2010, 3: 25-27 [张梅军, 李曙光, 孙启亮. 四种典型时频分析方法研究与仿真. 仪器仪表与分析监测, 2010, 3: 25-27]

3 Bogaert C, Beckers F, Ramaekers D, et al. Analysis of heart rate variability with correlation dimension method in a normal population and in heart transplant patients. Autonomic Neurosci, 2001, 90: 142-147

4 Eckmann J P, Oliffson Kamphorst S, Ruelle D, et al. Liapunov exponents from time series. Phys Rev A, 1986, 34: 4971-4979

5 Richman J S, Moorman J R. Physiological time-series analysis using approximate entropy and sample entropy. Am J Physiol Heart Circ Physiol, 2000, 278: H2039-H2049

6 Berger S, Schneider G, Kochs E F, et al. Permutation entropy: Too complex a measure for EEG time series? Entropy, 2017, 19: 692

7 Ivanov P C, Amaral L A N, Goldberger A L, et al. Multifractality in human heartbeat dynamics. Nature, 1999, 399: 461-465

8 Fiskum C, Andersen T G, Bornas X, et al. Non-linear heart rate variability as a discriminator of internalizing psychopathology and negative affect in children with internalizing problems and healthy controls. Front Physiol, 2018, 9: 561

9 Zhao H, He S. Analysis of speech signals' characteristics based on MF-DFA with moving overlapping windows. Phys A-Stat Mech Appl, 2016, 442: $343-349$

10 Wang F, Fan Q, Stanley H E. Multiscale multifractal detrended-fluctuation analysis of two-dimensional surfaces. Phys Rev E, 2016, 93: 042213

11 Turiel A, Pérez-Vicente C J, Grazzini J. Numerical methods for the estimation of multifractal singularity spectra on sampled data: A comparative study. J Comput Phys, 2006, 216: 362-390

12 Marwan N, Wessel N, Meyerfeldt U, et al. Recurrence-plot-based measures of complexity and their application to heart-rate-variability data. Phys Rev E, 2002, 66: 026702

13 Zhang J, Small M. Complex network from pseudoperiodic time series: Topology versus dynamics. Phys Rev Lett, 2006, 96: 238701

14 Shao Z G. Network analysis of human heartbeat dynamics. Appl Phys Lett, 2010, 96: 073703

15 Li X, Dong Z. Detection and prediction of the onset of human ventricular fibrillation: An approach based on complex network theory. Phys Rev E, 2011, 84: 062901

16 Donner R V, Small M, Donges J F, et al. Recurrence-based time series analysis by means of complex network methods. Int J Bifurcation Chaos, 
2011, 21: 1019-1046

17 Budroni M A, Baronchelli A, Pastor-Satorras R. Scale-free networks emerging from multifractal time series. Phys Rev E, 2017, 95: 052311

18 Jalan S, Yadav A, Sarkar C, et al. Unveiling the multi-fractal structure of complex networks. Chaos Soliton Fract, 2017, 97: 11-14

19 Lacasa L, Just W. Visibility graphs and symbolic dynamics. Phys D-Nonlinear Phenom, 2018, 374-375: 35-44

20 Nunes Amaral L A, Ivanov P C, Aoyagi N, et al. Behavioral-independent features of complex heartbeat dynamics. Phys Rev Lett, 2001, 86: 60266029

21 López P, Tarquis A M, Matulka A, et al. Multiscaling properties on sequence of turbulent plumes images. Chaos Soliton Fract, 2017, 105: 128-136

22 Yao B, Imani F, Sakpal A S, et al. Multifractal analysis of image profiles for the characterization and detection of defects in additive manufacturing. J Manuf Sci Eng, 2018, 140: 031014

23 Liu Y, Ding D X. Nonlinear characteristics of electrocardiograph signals based on fractal (in Chinese). J Northwestern Polytech Univ, 2018, $36: 287$ -293 [刘雁, 丁冬晓. 基于分形的心电信号非线性特征研究. 西北工业大学学报, 2018, 36: 287-293]

24 Goldberger A L, Amaral L A N, Glass L, et al. PhysioBank, PhysioToolkit, and PhysioNet: Components of a new research resource for complex physiologic signals. Circulation, 2000, 101: e215-e220

25 Wang J, Ning X, Chen Y. Multifractal analysis of electronic cardiogram taken from healthy and unhealthy adult subjects. Phys A-Stat Mech Appl, 2003, 323: 561-568

26 Yang X D, He A J, Zhou Y, et al. Multifractal mass exponent spectrum of complex physiological time series. Chin Sci Bull, 2010, 55: 1996-2003

27 Chhabra A, Jensen R V. Direct determination of the $f(\alpha)$ singularity spectrum. Phys Rev Lett, 1989, 62: 1327-1330

28 Halsey T C, Jensen M H, Kadanoff L P, et al. Fractal measures and their singularities: The characterization of strange sets. Phys Rev A, 1986, 33: 1141-1151

29 Yang X, Ning X, Wang J. Multifractal analysis of human synchronous 12-lead ECG signals using multiple scale factors. Physica A-Stat Mech Appl, 2007, 384: 413-422

30 Wang J, Ning X, Ma Q, et al. Multiscale multifractality analysis of a 12-lead electrocardiogram. Phys Rev E, 2005, 71: 062902

31 Ning X, Xu Y, Wang J, et al. Approximate entropy analysis of short-term HFECG based on wave mode. Phys A-Stat Mech Appl, 2005, 346: 475483

32 Pan W. Application of decision tree to identify abnormal high frequency electro-cardiograph (in Chinese). Phys Exp, 2009, 29: 29-34 [潘雯. 基于 决策树的异常高频心电图识别. 物理实验, 2009, 29: 29-34]

$33 \mathrm{Xu}$ Y, Ning X, Chen Y, et al. Mode entropy and dynamical analysis of irregularity for HFECG. Chin Sci Bull, 2004, 49: 1886-1890

34 Goldberger A L, Amaral L A N, Hausdorff J M, et al. Fractal dynamics in physiology: Alterations with disease and aging. Proc Natl Acad Sci USA, 2002, 99: 2466-2472

35 Wang Z, Ning X, Zhang Y, et al. Distribution of correlation dimensions of synchronous 12-lead ECG signals. Chin Sci Bull, 2000, 45: 1628-1632

36 Wang Z. Lyapunov exponents for synchronous 12-lead ECG signals. Chin Sci Bull, 2002, 47: 1845-1848 


\title{
The complexity of ECG signal based on multifractal theories and its nonlinear dynamical mechanism
}

\author{
Xiaodong Yang ${ }^{1}$, Xuesong Wang $^{2 *}$, Aijun $\mathrm{He}^{3}$, Zhixiao Wang ${ }^{1} \&$ Jun Wang ${ }^{4}$ \\ ${ }^{1}$ School of Computer Science and Technology, China University of Mining and Technology, Xuzhou 221116, China; \\ ${ }^{2}$ Xuzhou Key Laboratory of Artificial Intelligence and Big Data, School of Information and Control Engineering, China University of Mining and \\ Technology, Xuzhou 221116, China; \\ ${ }^{3}$ School of Electronic Science and Engineering, Nanjing University, Nanjing 210023, China; \\ ${ }^{4}$ School of Geographic and Biologic Information, Nanjing University of Posts and Telecommunications, Nanjing 210023, China \\ * Corresponding author, E-mail: wangxuesongcumt@163.com
}

The nonlinear dynamical analysis of biomedical signals has always been the research focus in academic fields. It better uncovers the essential rules in life activities than conventional linear and time-frequency analysis methods. Human body surface electrocardiogram (ECG) signal is non-stationary and frequency-varying by nature, which belongs to a typical nonlinear signal. Therefore, traditional linear analysis methods cannot completely disclose its nonlinear nature. The existing nonlinear dynamic tools, such as correlation dimension $\left(\mathrm{D}_{2}\right)$, Lyapunov exponent, entropy, detrended fluctuation analysis, multifractal singularity spectrum etc., have already been applied to the studies on heart electrical signals, which proved that this signal has definite chaotic characteristic. The aforementioned nonlinear methods perform better in the analysis of deterministic stochastic signals.

In our viewpoints, because all the probabilities in the original data set can be exactly expressed by the corresponding points in the multifractal singularity spectrum $f(\alpha)$, the area of the spectrum must comprise the total information of the data. This two-dimensional expression is more accurate than the computation of spectral width $\Delta \alpha$ in analyzing the heartbeat signal. With regard to ECG time series, the areas of singularity spectrum should include all the information about the heartbeat dynamics. Considering the irregularity and anisotropy of the heartbeat electrical signal propagation, we investigated the arithmetic mean and dispersing degree of the area calculated from synchronous 12-lead ECG signals obtained from a large number of subjects, who were under different physiological and pathological conditions. The 12-lead ECG records were obtained from 12 sensors positioned on body surfaces, including the limb and chest leads. In addition, the ECG signals were in high frequency section (HFECG), whose frequency components were above $100 \mathrm{~Hz}$ (approximately $100 \mathrm{~Hz}-2 \mathrm{kHz}$ ). Some early heart diseases can first be reflected by high-frequency ECG signals, which are often associated with sharp notches and slurs in the time domain. Furthermore, we set sampling frequency of HFECG signal to $1 \mathrm{kHz}$ so that all the components below $500 \mathrm{~Hz}$ could be analyzed according to the sampling theorem.

Thereafter, by the virtue of the multifractal theory, we investigated the arithmetic mean and dispersing degree computed from singularity spectrum area of synchronous 12-lead ECG signals obtained from different crowds of human subjects. Variance analysis tests revealed all 12lead ECG signals from above cohorts were statistically identifiable using these two indicators. The experimental results suggest that the arithmetic mean of the area of the 12-lead ECG signals was apparently large for healthy young but small for myocardial infarction (MI) sufferers. Besides, the dispersing degree of the area of the 12-lead ECG signals was obviously small for healthy young but large for MI sufferers. For the other cohorts, such as ischemia sufferers and healthy elderly, these two values were of middle magnitudes. This indicates that with deeper lesions, the fractal-like structure of the heartbeat system is damaged or structurally changed, which may lead to decline in the nonlinear complexity of the system and concomitant increase in the irregularity and anisotropic propagation of ECG signal. In addition, we found that the 12-lead mean value of singularity spectrum area of human ECG signals can reflect the self-discipline control status of human autonomic nerve to some degree. This value gradually decreased with aging. These findings suggest that the self-discipline control of the human autonomic nervous system weakens with aging. The nonlinear complexity of ECG signal then descends and tends to turn from multifractality to monofractality, implying weakened human individual adaptabilities. Our studies on the nonlinear dynamical features of heart electrical signals and ECG variations with age, disease, and human autonomic nerve control, demonstrate theoretical and diagnostic significances.

electrocardiogram (ECG), multifractal singularity spectrum, arithmetical mean, dispersing degree

doi: 10.1360/N972019-00235 